\title{
Phase transition dynamics for baryon-dense matter
}

\author{
Jørgen Randrup* \\ Nuclear Science Division, Lawrence Berkeley National Laboratory, Berkeley, California 94720 \\ E-mail: JRandrup@LBL.gov
}

The dynamical description of the onset and development of the phase separation caused by a firstorder phase transition requires a transport model that incorporates finite-range effects. Here we introduce a gradient term in the compressional energy of a simple two-phase equation of state and apply the resulting model to the interface between the two coexisting phases. Subsequently, within the framework of both ideal and viscous fluid dynamics, we derive the dispersion relations for the collective modes in bulk matter and make illustrative calculations of the corresponding spinodal growth rates. Finally, we discuss the prospects for spinodal phase separation to occur in actual nuclear collisions.

5th International Workshop on Critical Point and Onset of Deconfinement - CPOD 2009,

June 08 - 122009

Brookhaven National Laboratory, Long Island, New York, USA

\footnotetext{
${ }^{*}$ Speaker.
} 


\section{Introduction}

The phase structure of strongly interacting matter presents a focal point for research in heavyion physics. Currently, RHIC at BNL is preparing for a beam energy scan that aims to identify signals of the expected critical point, while the CBM experiment at the future FAIR at GSI will explore the properties of compressed baryonic matter and search for the expected first-order phase transition, and JINR in Dubna is planning NICA for studying the mixed phase.

However, it will be no easy task to extract the thermodynamic phase structure from nuclear collision experiments. In addition to the inherent problems arising from the smallness of the collision system (which renders its spatial configuration far from uniform) and its rapid evolution (which prevents global equilibrium from being established), the experimentalist is faced with the problem that there exists yet no suitable dynamical model with which to simulate the collisions for the purpose of anticipating the observable effects of the phase structure.

A particularly urgent task is the identification of suitable observables that can signal the existence of a phase transition. The present work is motivated by possibility that the spinodal instabilities that invariably accompany a first-order phase structure may cause the bulk of the collision system to clump and thereby generate extractable correlations with sufficient specificity, in analogy to spinodal nuclear multifragmentation $[1,2]$. While some explorations of possible experimental signals have already been made $[3,4,5,6]$, their practical utility is hampered by the lack of dynamical transport models that encompass the essential phase-transition physics. It is therefore not possible to assess the degree to which those various effects may actually develop in a collision setting. Nor has it been determined whether they would in fact survive the post-hadronization expansion stage in an observable form.

An ideal transport model would explicitly evolve the microscopic degrees of freedom in the system, which change from being partonic in the deconfined sector to being hadronic in the confined sector. Such a description has not yet been developed, even for static scenarios, and one must rely on less fundamental descriptions. While a variety of transport models have achieved considerable success in reproducing observables for high-energy collisions over a range of energies, they need further development before they can be applied to phase transition dynamics. Roughly speaking, the current microscopic transport models do not yet contain a phase transition, while the fluid-dynamical models are spatially local and therefore unable to capture the key feature of the spinodal phenomenon, namely the spontaneous emergence of a characteristic length scale [7]. We illustrate here the particular importance of incorporating a finite range into the treament.

The presentation is organized as follows. First we construct a schematic two-phase equation of state to serve as a concrete framework for our discussions. We then introduce a gradient term in the compressional energy and derive the corresponding generalized thermodynamics. On this basis, we consider the equilibium interface between the two coexisting phases and derive both the associated interface tension and the density profile. Subsequently, within the framework of fluid dynamics, we consider harmonic distortions in uniform matter and derive the corresponding dispersion relation for both ideal and viscous scenarios; we discuss especially the spinodal phase regiuon where such modes are amplified exponentially. Finally, we discuss in a qualitative manner the strategy for achieving spinodal phase separation in actual nuclear collisions. A more detailed discussion can be found in Ref. [8]. 


\section{Equation of state}

For the purpose of making numerical illustrations, we adopt a schematic model that is a generalization of a classical gas in a density-dependent mean field. The resulting equation of state has certain generic deficiencies and the results should therefore not be taken at face value. The model assumes that the energy density $\varepsilon$ is the sum of a compressional term $w_{0}(\rho)$, where $\rho$ is the (net) baryon density, and a thermal term, $\kappa=\frac{d}{2} \rho T$, where $d$ is an adjustable parameter. Denoting the entropy density of the corresponding generalized ideal classical gas by $\sigma(\kappa, \rho)$, we may write the entropy density as a function of $\varepsilon$ and $\rho$ as $\sigma(\varepsilon, \rho)=\delta\left(\varepsilon-w_{0}(\rho), \rho\right)$. The associated Lagrange coefficients are then $\beta(\varepsilon, \rho)=\partial_{\varepsilon} \sigma(\varepsilon, \rho)=1 / T(\varepsilon, \rho)$ and $\alpha(\varepsilon, \rho)=\partial_{\rho} \sigma(\varepsilon, \rho)=-\mu(\varepsilon, \rho) / T(\varepsilon, \rho)$.

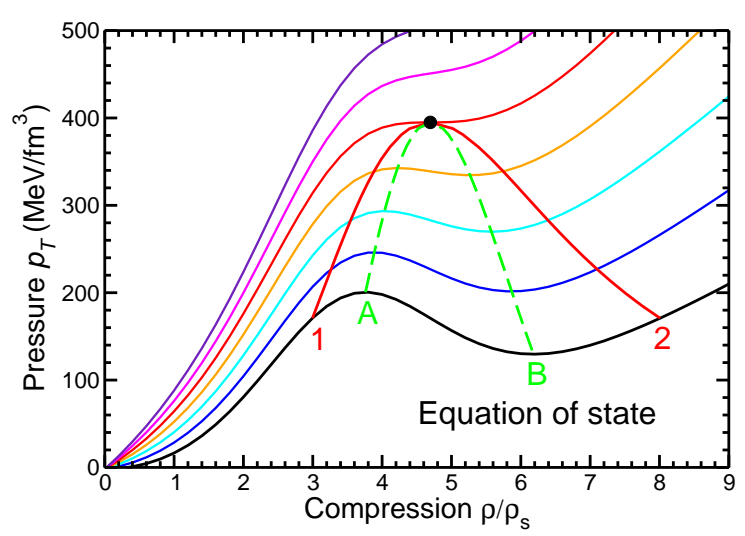

Figure 1: The equation of state $p_{T}(\rho)$ : The pressure $p$, as obtained with the schematic model, shown for a number of temperatures, $T / T_{c}=0, \frac{1}{4}, \frac{1}{2}, \frac{3}{4}, 1, \frac{5}{4}, \frac{3}{2}$, as a function of the density $\rho$. The phase coexistence (solid) and the spinodal (dashed) boundaries are indicated; they coincide at the critical point (dot). The pressure has extrema at the spinodal boundaries and so decreases in between, rendering the matter mechanically unstable.

By interpolating between a hadron gas and a quark-gluon plasma it is possible to adjust $w_{0}(\rho)$ so that those two phases coexist at $T=0$. The resulting equation of state $p_{T}(\rho)$ is shown in Fig. 1 , with the critical point as well as the regions of phase coexistence and spinodal instability indicated. Figure 2 shows the corresponding phase diagram using either $\rho$ or $\mu$, together with a number of isentropic phase trajectories. The density is more convenient in the presence of a phase transition.
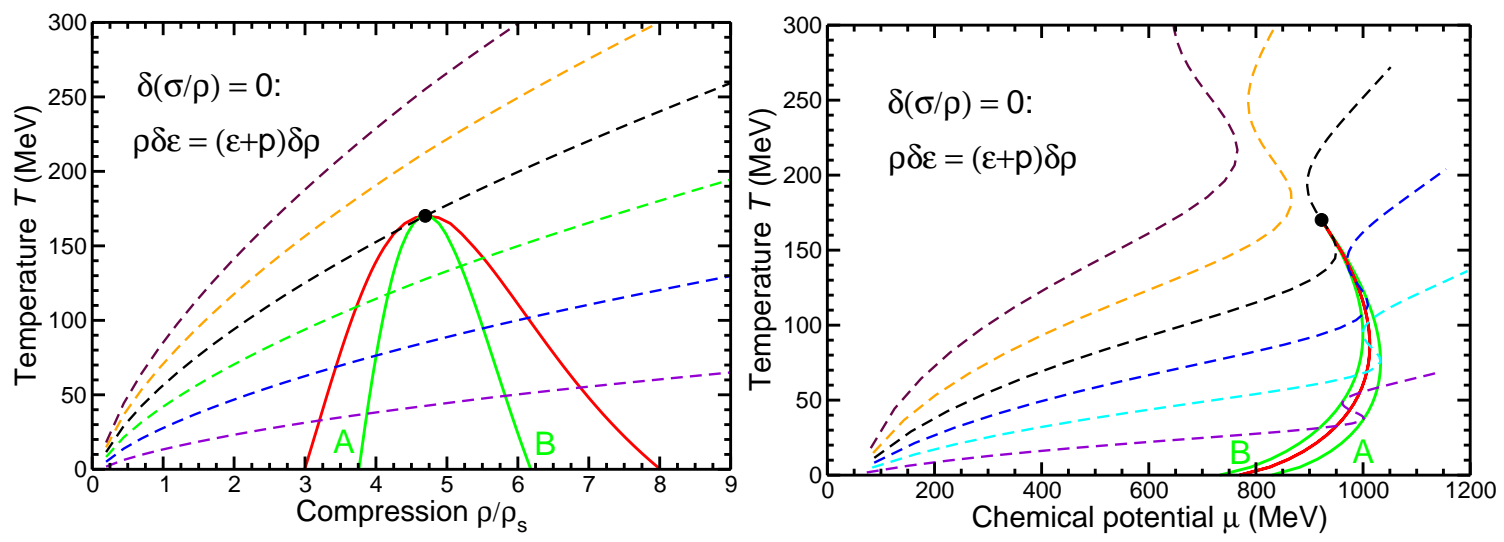

Figure 2: The phase diagram implied by the equation of state shown in Fig. 1 in the $\rho-T$ (left) and the $\mu-T$ (right) plane, together with several isentropic trajectories (dashed curves). Changing from $\rho$ to $\mu$ causes the two coexisting phase points (red curve) to coincide, while the phase trajectories exhibit significant contortions that extend between the two spinodal boundaries (green curve) below the critical temperature. 


\section{Gradient corrections}

As a simple way to take approximate account of finite range effects, we employ a gradient correction in the compressional energy and write the local interaction-energy density on the form

$$
\tilde{w}(\mathbf{r})=w_{0}(\tilde{\rho}(\mathbf{r}))+\frac{1}{2} C(\nabla \tilde{\rho}(\mathbf{r}))^{2}=w_{0}(\tilde{\rho}(\mathbf{r}))+\frac{1}{2} a^{2} \varepsilon_{\mathrm{g}}\left(\frac{\nabla \tilde{\rho}(\mathbf{r})}{\rho_{\mathrm{g}}}\right)^{2} .
$$

The strength of the gradient term has the form $C=a^{2} \varepsilon_{\mathrm{g}} / \rho_{\mathrm{g}}^{2}$, where $\rho_{\mathrm{g}}$ is a (baryon) charge density and $\varepsilon_{\mathrm{g}}$ is an energy density. We choose the phase point $\left(\rho_{\mathrm{g}}, \varepsilon_{\mathrm{g}}\right)$ to be in the middle of the phase coexistence region, $\rho_{\mathrm{g}} \doteq \rho_{c}=4.70 \rho_{0}$ and $\varepsilon_{\mathrm{g}} \doteq \varepsilon_{T=T_{c} / 2}\left(\rho_{c}\right)=\frac{1}{2}\left(w_{0}\left(\rho_{c}\right)+\varepsilon_{c}\right)=561 \mathrm{MeV} / \mathrm{fm}^{3}$. The gradient term is then governed by the length $a$ which we consider to be somewhat adjustable. (A gradient term was also used in recent fluid dynamic studies of the hadron-quark transition [9].)

In order to derive the corresponding thermodynamic relations, we assume that the entropy density still has the form $\tilde{\sigma}(\mathbf{r})=\delta(\tilde{\kappa}(\mathbf{r}), \tilde{\rho}(\mathbf{r}))=\delta(\tilde{\varepsilon}(\mathbf{r})-\tilde{w}(\mathbf{r}), \tilde{\rho}(\mathbf{r}))$, where $\tilde{\kappa}(\mathbf{r})=\tilde{\varepsilon}(\mathbf{r})-\tilde{w}(\mathbf{r})$ is the local thermal density. The local Lagrange coefficients can then be obtained from a variation of the total entropy $S[\tilde{\varepsilon}(\mathbf{r}), \tilde{\rho}(\mathbf{r})]=\int d \mathbf{r} \tilde{\sigma}(\mathbf{r})$ with respect to $\tilde{\varepsilon}(\mathbf{r})$ and $\tilde{\rho}(\mathbf{r}), \tilde{\beta}(\mathbf{r})=\delta S / \delta \tilde{\varepsilon}(\mathbf{r})$ and $\tilde{\alpha}(\mathbf{r})=\delta S / \delta \tilde{\rho}(\mathbf{r})$, yielding an explicit gradient term in the chemical potential $\tilde{\mu}(\mathbf{r})$. The resulting expression for the local pressure is then

$$
\tilde{p}(\mathbf{r})=\tilde{\sigma}(\mathbf{r}) \tilde{T}(\mathbf{r})-\tilde{\varepsilon}(\mathbf{r})+\tilde{\mu}(\mathbf{r}) \tilde{\rho}(\mathbf{r})+C(\nabla \tilde{\rho}(\mathbf{r}))^{2},
$$

It follows that $\nabla(\tilde{p}(\mathbf{r}) / \tilde{T}(\mathbf{r}))=-\tilde{\varepsilon}(\mathbf{r}) \nabla \tilde{\beta}(\mathbf{r})-\tilde{\rho}(\mathbf{r}) \nabla \tilde{\alpha}(\mathbf{r})$, which can be regarded as a generalization of the familiar bulk relation $\delta(p / T)=-\varepsilon \delta \beta-\rho \delta \alpha$; it ensures that $\tilde{p}(\mathbf{r})$ will be constant whenever $\tilde{T}(\mathbf{r})$ and $\tilde{\mu}(\mathbf{r})$ are. We also note that the gradient correction (3.1) to the compressional energy density migrates directly into the local free energy density,

$$
\tilde{f}_{T}(\mathbf{r})=\kappa_{T}(\tilde{\rho}(\mathbf{r}))+\tilde{w}(\mathbf{r})-T \delta\left(\kappa_{T}(\tilde{\rho}(\mathbf{r})), \tilde{\rho}(\mathbf{r})\right)=f_{T}(\tilde{\rho}(\mathbf{r}))+\frac{1}{2} C(\nabla \tilde{\rho}(\mathbf{r}))^{2} .
$$

\section{Interface equilibrium}

Once the finite-range effects have been included, we may treat the interface between the two coexisting phases. We denote the coexistence values of temperature, chemical potential, and pressure by $T_{0}, \mu_{0}$, and $p_{0}$. and assume that the two systems have a planar interface perpendicular to the $x$ direction. Global equilibrium requires that the total entropy $S$ be constant under variations $\delta \tilde{\varepsilon}(x)$ and $\delta \tilde{\rho}(x)$ that conserve the total energy $E=\int d x \tilde{\varepsilon}(x)$ and the total (net) charge $B=\int d x \tilde{\rho}(x)$,

$$
0 \doteq \delta S-\beta_{0} \delta E-\alpha_{0} \delta B=\int d x\left\{\left[\tilde{\beta}(x)-\beta_{0}\right] \delta \tilde{\varepsilon}(x)+\left[\tilde{\alpha}(x)-\alpha_{0}\right] \delta \tilde{\rho}(x)\right\}
$$

thus implying spatial constancy of temperature and chemical potential, $\tilde{\beta}(x) \doteq \beta_{0}$ and $\tilde{\alpha}(x) \doteq \alpha_{0}$.

Because the temperature is constant we may work in the canonical framework and proceed as Ravenhall et al. [10]. Since $\partial_{\rho} f_{T}(\rho)=\mu_{T}(\rho)$, the condition for equilibrium is then expressed as

$$
0 \doteq \delta \int d x\left[\tilde{f}_{T_{0}}(x)-\mu_{0} \tilde{\rho}(x)\right]=\int d x\left[\mu_{T_{0}}(\tilde{\rho}(x))-C \partial_{x}^{2} \tilde{\rho}(x)-\mu_{0}\right] \delta \tilde{\rho}(x)
$$



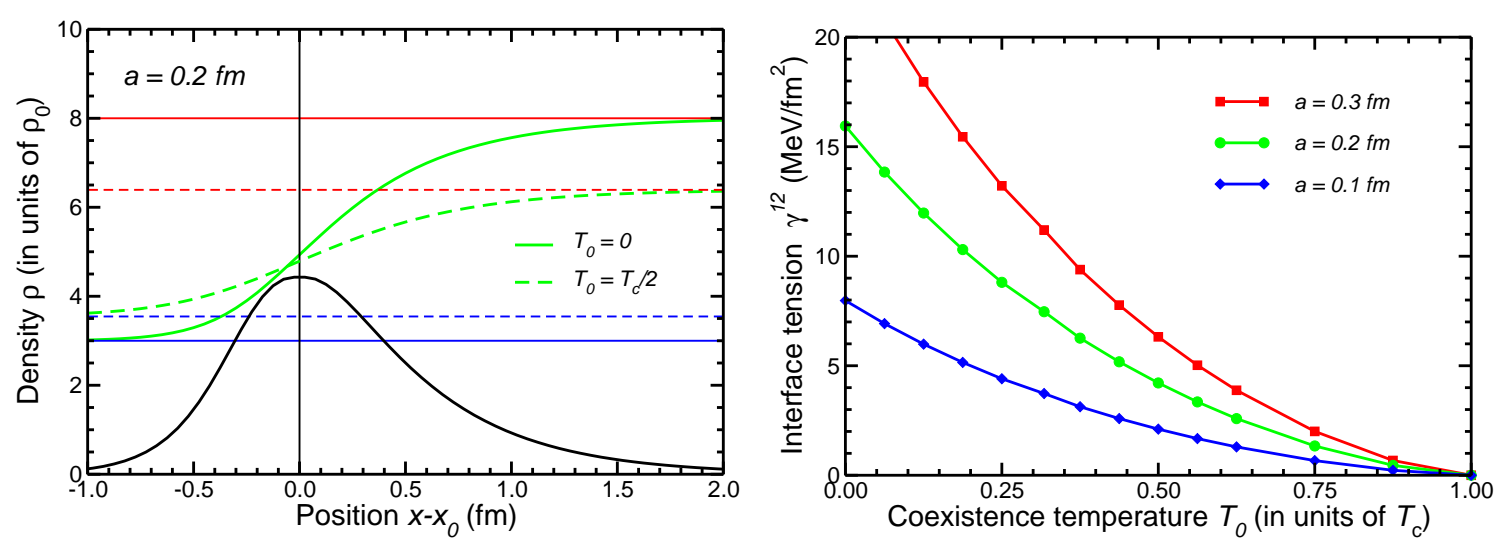

Figure 3: Left: The surface profile $\tilde{\rho}(x)$ for $T=0$ (solid) and $T=\frac{1}{2} T_{c}$ (dashed), using as a reference for $x$ the position $x_{0}$ where the chemical potential equals the coexistence value of the bulk chemical potential, $\mu_{T}\left(\tilde{\rho}\left(x=x_{0}\right)\right) \doteq \mu_{0}(T)$. The limiting (coexistence) densities are shown by the horizonthal lines, while the bottom curve is the interface location function $g(x)=\partial_{x} \tilde{\rho}(x) / \rho_{21}$ [11] for $T=0$. Right: The specific interface tension $\gamma_{T_{0}}^{12}[12]$ as a function of the coexistence temperature $T_{0}$ for various values of the range $a$.

Thus the equilibrium profile $\tilde{\rho}(x)$ is determined by the following differential equation,

$$
C \partial_{x}^{2} \tilde{\rho}(x) \doteq \mu_{T_{0}}(\tilde{\rho}(x))-\mu_{0}=\partial_{\rho} \Delta f_{T_{0}}(\tilde{\rho}(x)),
$$

where $\Delta f_{T_{0}}(\rho)$ is the difference between the free energy density of a uniform system of density $\rho$, $f_{T_{0}}(\rho)$, and the "Maxwell" free energy density associated with the corresponding phase mixture, $f_{T_{0}}^{M}(\rho) \equiv f_{T_{0}}\left(\rho_{i}\right)+\mu_{0}\left(\rho-\rho_{i}\right) \leq f_{T_{0}}(\rho)$ where $\rho_{i}$ is either one of the two coexistence densities. The density profile is then given by

$$
\tilde{\rho}(x)=\tilde{\rho}\left(x_{0}\right)+\rho_{c} \int_{x_{0}}^{x}\left[\frac{2}{\varepsilon_{g}} \Delta f_{T_{0}}(\tilde{\rho}(x))\right]^{\frac{1}{2}} \frac{d x}{a},
$$

where $x_{0}$ is some location where the density is known, for example the location where $\Delta f_{T_{0}}(\tilde{\rho}(x))$ has its maximum and hence $\mu_{T_{0}}\left(\tilde{\rho}\left(x_{0}\right)\right)=\mu_{0}$. The resulting density profile is illustrated in Fig. 3 .

The local excess in the free energy density due to the interface is given by

$$
\tilde{f}_{T_{0}}^{12}(x)=\tilde{f}_{T_{0}}(x)-f_{T_{0}}^{M}(\tilde{\rho}(x))=\Delta f_{T_{0}}(\tilde{\rho}(x))+\frac{1}{2} C\left(\partial_{x} \tilde{\rho}(x)\right)^{2}=2 \Delta f_{T_{0}}(\tilde{\rho}(x)) .
$$

so the total deficit in free energy per unit interface area, the interface tension [12], is given by

$$
\gamma_{T_{0}}^{12}=\int_{-\infty}^{+\infty} d x \tilde{f}_{T_{0}}^{12}(x)=2 \int \frac{d \tilde{\rho}(x)}{\partial_{x} \tilde{\rho}(x)} \Delta f_{T_{0}}(\tilde{\rho}(x))=a \int_{\rho_{1}}^{\rho_{2}} \frac{d \rho}{\rho_{\mathrm{g}}}\left[2 \varepsilon_{\mathrm{g}} \Delta f_{T_{0}}(\rho)\right]^{\frac{1}{2}} .
$$

This quantity can be obtained without explicit knowledge of the profile $\tilde{\rho}(x)$ and it scales directly with the length parameter $a$; it is shown in Fig. 3 as a function of temperature. It decreases steadily from its maximum value at $T=0$ until it vanishes at $T_{c}$. With the (somewhat arbitrary) parameter values adopted, the zero-temperature interface tension is $\gamma_{0}^{12} \approx 16 \mathrm{MeV} / \mathrm{fm}^{3}$, about 16 times the familiar nuclear surface tension. This value lies near the lower end of the rather wide range of expected values for the tension between quark and nuclear matter (typical low values are $10-$ $20 \mathrm{MeV} / \mathrm{fm}^{2}$, while typical high values are $50-100 \mathrm{MeV} / \mathrm{fm}^{2}$, see for example Refs. [13, 14]). 


\section{Collective modes}

The gradient correction affects the dynamical response to small density undulations imposed on static and uniform matter, $\delta \varepsilon(\mathbf{r})=\tilde{\varepsilon}(\mathbf{r})-\bar{\varepsilon}$ and $\delta \rho(\mathbf{r})=\tilde{\rho}(\mathbf{r})-\bar{\rho}$. (The local change in the pressure is then of a similar form, $\delta p(\mathbf{r})=\tilde{p}(\mathbf{r})-\bar{p}$ with $\bar{p}=p(\bar{\varepsilon}, \bar{\rho})$.) Assuming (at first) that the time evolution is described by ideal fluid dynamics, the equations of motion then arise from energymomentum conservation, $\partial_{\mu} T^{\mu \nu}=0$, together with conservation of (baryon) charge, $\partial_{\mu} j^{\mu}=0$.

Assuming that the local flow velocities $\mathbf{v}(\mathbf{r})$ are non-relativistic, we may ignore $v^{2}$ and thus put $\gamma$ to unity. This yields the following five equations of motion,

$$
\begin{aligned}
& 0=\partial_{\mu} T^{\mu 0}(\mathbf{r}, t) \approx \partial_{t} \delta \varepsilon+\bar{h} \partial_{i} v^{i} \\
& 0=\partial_{\mu} T^{\mu i}(\mathbf{r}, t) \approx \bar{h} \partial_{t} v^{i}+\partial^{i} \delta p \\
& 0=\partial_{\mu} j^{\mu}(\mathbf{r}, t) \approx \partial_{t} \delta \rho+\bar{\rho} \partial_{i} v^{i}
\end{aligned}
$$

where $\bar{h}=\bar{\varepsilon}+\bar{p}$ is the enthalpy density of the uniform system. As usual, the equations for $T^{\mu v}$ can be combined to a sound-wave equation, while a comparison of the first and last equations yields the evolution of the density disturbance in terms of that of the energy disturbance, so

$$
\partial_{t}^{2} \delta \varepsilon(\mathbf{r})=\nabla^{2} \delta p(\mathbf{r}), \bar{h} \partial_{t} \delta \rho(\mathbf{r})=\bar{\rho} \partial_{t} \delta \varepsilon(\mathbf{r}) .
$$

It is straightforward to see that, to leading order in the disturbances $\delta \varepsilon(\mathbf{r})$ and $\delta \rho(\mathbf{r})$, the local pressure is $\tilde{p}(\mathbf{r}) \approx p(\tilde{\varepsilon}(\mathbf{r}), \tilde{\rho}(\mathbf{r}))-C \bar{\rho} \nabla^{2} \rho(\mathbf{r})$. The first term is the usual local-density approximation, i.e. the pressure is calculated as in uniform matter that has been prepared with the local density values, while the second term arises from the gradient correction to the chemical potential. Therefore, to the same order, we have

$$
\nabla^{2} \delta p(\mathbf{r}) \approx p_{\varepsilon} \nabla^{2} \varepsilon(\mathbf{r})+p_{\rho} \nabla^{2} \rho(\mathbf{r})-C \bar{\rho} \nabla^{4} \rho(\mathbf{r})
$$

where $p_{\varepsilon} \equiv \partial_{\varepsilon} p(\varepsilon, \rho)$ and $p_{\rho} \equiv \partial_{\rho} p(\varepsilon, \rho)$ evaluated at the local phase point $(\varepsilon, \rho)=(\tilde{\varepsilon}(\mathbf{r}), \tilde{\rho}(\mathbf{r}))$.

For harmonic undulations, $\delta \varepsilon(\mathbf{r})=\varepsilon_{\mathbf{k}} \exp (i \mathbf{k} \cdot \mathbf{r}-i \omega t)$ and $\delta \rho(\mathbf{r})=\rho_{\mathbf{k}} \exp (i \mathbf{k} \cdot \mathbf{r}-i \omega t)$, the continuity equation requires $\bar{h} \rho_{\mathbf{k}}=\bar{\rho} \varepsilon_{\mathbf{k}}$. The dispersion relation is then readily obtained from (5.4),

$$
\omega_{k}^{2}=v_{s}^{2} k^{2}+C \frac{\bar{\rho}^{2}}{\bar{h}} k^{4}=v_{s}^{2} k^{2}+a^{2} \frac{\varepsilon_{\mathrm{g}}}{\bar{h}} \frac{\bar{\rho}^{2}}{\rho_{\mathrm{g}}^{2}} k^{4} .
$$

with $v_{s}$ being the isentropic speed of sound,

$$
v_{s}^{2}=\frac{\rho}{h}\left(\frac{\partial p}{\partial \rho}\right)_{s}=p_{\varepsilon}+\frac{\bar{\rho}}{\bar{h}} p_{\rho}=-\frac{\bar{T}}{\bar{h}}\left[\bar{h}^{2} \sigma_{\varepsilon \varepsilon}+2 \bar{h} \bar{\rho} \sigma_{\varepsilon \rho}+\bar{\rho}^{2} \sigma_{\rho \rho}\right],
$$

where $\sigma_{\varepsilon \rho} \equiv \partial_{\varepsilon} \partial_{\rho} \sigma(\varepsilon, \rho)$ evaluated at $(\varepsilon, \rho)=(\bar{\varepsilon}, \bar{\rho})$, etc.. The first term is what emerges in ordinary ideal fluid dynamics and it is perfectly linear, $\omega_{k}=v_{s} k$. That pathological behavior is modified by the gradient term which generally increases $\omega_{k}^{2}$. In the spinodal region, where $v_{s}^{2}$ is negative, the collective frequency is imaginary, $\omega_{k}= \pm i \gamma_{k}$, and the gradient term then suppresses the growth of high- $k$ modes. As a result, the growth rate $\gamma_{k}$ will exhibit a maximum followed by a rapid fall-off to zero as a function of the wave number $k$, as is familiar from other substances exhibiting spinodal instability [2,7]. (See Ref. [15] for an earlier approximate treatment of this.) 

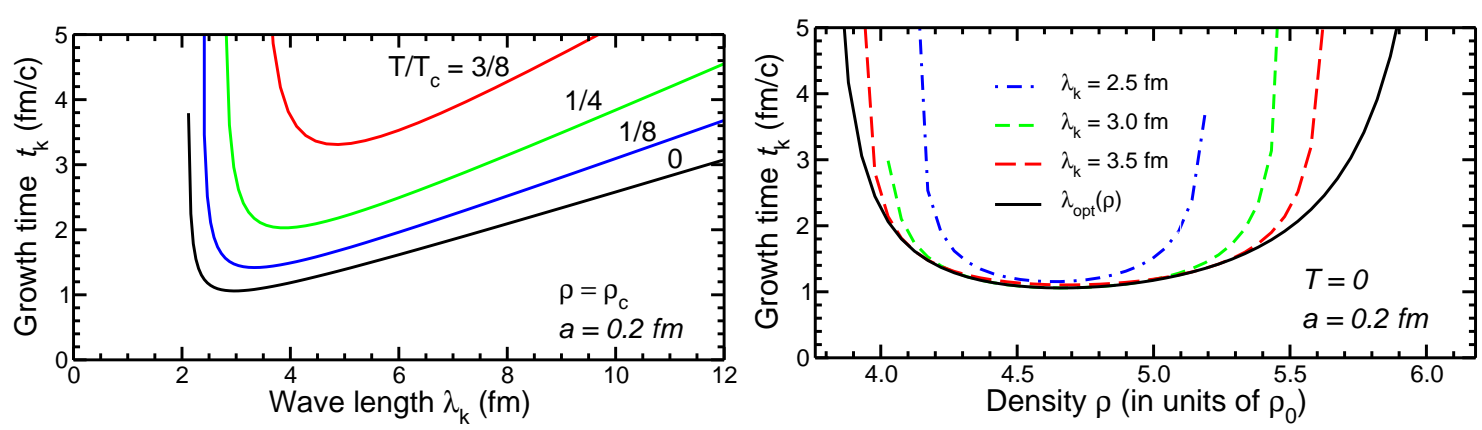

Figure 4: The growth times $t_{k}(\rho, T)=1 / \gamma_{k}$ vs. the wave length $\lambda_{k}=2 \pi / k$ for various temperatures $T$ at $\rho=\rho_{c}$ (left) and vs. the degree of compression, $\rho / \rho_{0}$, at $T=0$ for several values of $\lambda_{k}$ (right).

The spinodal growth rates $\gamma_{k}$ depend on the environment, specified for example by $\bar{\rho}$ and $\bar{T}$, as is illustrated in Fig. 4. Generally $\gamma_{k}(\bar{\rho}, \bar{T})$ vanishes along the spinodal boundary and it decreases as a function of temperature. With the present model we find that the fastest mode for $\bar{\rho}=\rho_{c}$ has a wave length of $\lambda_{\mathrm{opt}} \approx 3 \mathrm{fm}$ and a growth time of $t_{\mathrm{opt}} \approx 1.0 \mathrm{fm} / c$. As the temperature is raised, the maximum wave number $k_{\max }$ decreases as do the optimal values $k_{\text {opt }}$ and $\gamma_{\text {opt }}$. While the obtained temperature dependence is quite significant, it should be recognized that the thermal properties of the present model may not be realistic. On the other hand, the dependence of $\gamma_{k}(\bar{\rho}, \bar{T})$ on density is more moderate in the phase region of most rapid growth (as in dilute nuclear matter [2]).

It is important to appreciate that the phase region of instability for ideal fluid dynamics is bounded by the isentropic spinodal (where $v_{s}=0$ ) and it therefore lies inside the region of thermodynamic instabilty which is bounded by the isothermal spinodal. There are unstable isentropic modes whenever $v_{s}^{2}<0$. At zero temperature this amounts to $\partial_{\rho}^{2} w_{0}(\rho)<0$, which occurs exactly within the isothermal spinodal density region, as one would expect since $T=0 \Leftrightarrow \sigma=0$. However, as $T$ is increased, the region of isentropic instability shrinks faster than the region of isothermal instability and it disappears entirely at $T_{\max }=\frac{3}{5} T_{c}$ in the present model.

The above analysis was based on ideal fluid dynamics which conserves entropy, $\partial_{\mu} \sigma^{\mu}=0$, where $\sigma^{\mu}=\sigma u^{\mu}$ is the entropy current density. We now briefly discuss the effects of including viscosity into the fluid-dynamic treatment. Within the non-relativistic framework used for the derivation of the above dispersion relation, the inclusion of shear and bulk viscosity changes the pressure gradient by the term $-\nabla\left[\frac{4}{3} \eta+\zeta\right] \nabla \cdot \mathbf{v}$ where $\eta$ and $\zeta$ are the shear and bulk viscosity coefficients, respectively. The dispersion equation is then modified accordingly,

$$
\omega^{2}=v_{s}^{2} k^{2}+a^{2} \frac{\varepsilon_{\mathrm{g}}}{\bar{h}} \frac{\bar{\rho}^{2}}{\rho_{\mathrm{g}}^{2}} k^{4}-i\left[\frac{4}{3} \eta+\zeta\right] \frac{k^{2}}{\bar{h}} \omega
$$

where we have assumed that the combination $\xi \equiv \frac{4}{3} \eta+\zeta$ can be regarded as constant, for simplicity. Clearly, the zero-frequency modes occur for the same wave numbers as before, $k=0$ and $k=k_{\max }$, and the inclusion of viscosity does not afffect the location of the spinodal boundary. (However, if thermal conductivity were included, the spinodal boundary would gradually expand towards the isothermal boundary [10].) To leading order, the viscosity adds a negative imaginary term to the frequency, $-\frac{i}{2} \xi k^{2} / \bar{h}$, giving rise to an exponential damping factor. Furthermore, inside 
the spinodal region the collective frequencies are still purely imaginary, $\omega=i \gamma_{ \pm}$, and we find

$$
\gamma_{ \pm}= \pm\left[\left|v_{s}^{2}\right| k^{2}-a^{2} \frac{\varepsilon_{\mathrm{g}}}{\bar{\varepsilon}} \frac{\bar{\rho}^{2}}{\rho_{\mathrm{g}}^{2}} k^{4}+\frac{1}{4} \xi^{2} \frac{k^{4}}{\bar{h}^{2}}\right]^{\frac{1}{2}}-\frac{1}{2} \xi \frac{k^{2}}{\bar{h}}
$$

Thus the growth rate $\gamma_{+}$is reduced by $\approx \frac{1}{2} \xi k^{2} / \bar{h}$ and the optimal wave number becomes smaller as well. Even though the qualitative features will remain the same, the viscous effects may be quantitatively important [9].

In order to illustrate the key role played by the finite range in producing spinodal decomposition, let us briefly consider what would happen without the gradient term. We already noted that the resulting non-viscous dispersion relation would exhibit linear growth, $\gamma_{k}=\left|v_{s}^{2}\right| k$, and thus not favor any particular length scale. When viscosity is included, the growth rate would still grow monotonically, $\partial_{k} \gamma_{+}(k)>0$, but level off for large $k$,

$$
\gamma_{+}(k \rightarrow \infty) \approx \frac{\bar{\rho}}{\xi}\left|v_{s}^{2}\right|\left[1-\frac{3}{4} \frac{\bar{\rho}^{2}}{\xi^{2}} \frac{\left|v_{s}^{2}\right|}{k^{2}}+\ldots\right] .
$$

Thus the large- $k$ divergence characteristic of standard ideal fluid dynamics would be eliminated, but there would still not be a preferred length scale.

\section{Spinodal decomposition?}

In order to understand under what experimental conditions spinodal decomposition may actually occur, we must consider how the thermodynamic conditions in the bulk of the collision system evolve in the course of time. Such phase trajectories were studied for gold-gold collisions with a variety of dynamical transport models [16], and two examples are shown in Fig. 5. These results are useful for estimating which phase regions are being explored at given collisions energies and, in particular, how much time is being spent in the various unstable phase regions.
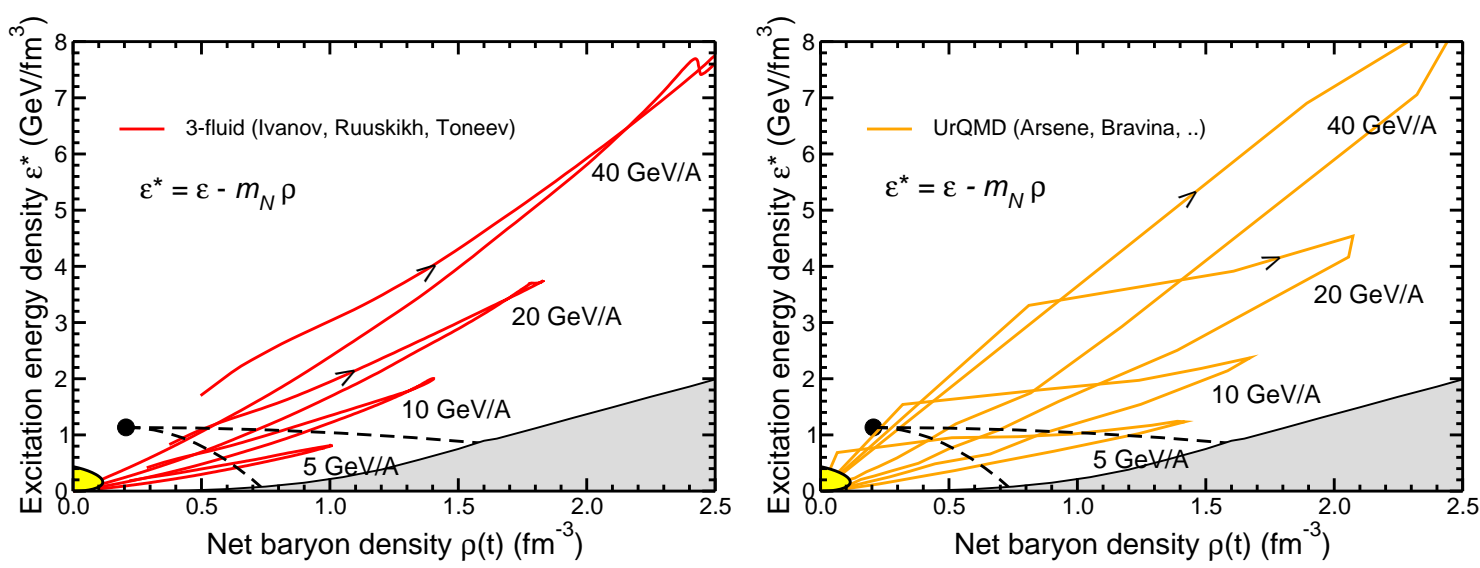

Figure 5: The combined time evolution of the energy density $\varepsilon$ and the net baryon density $\rho$ in the center of a $\mathrm{Au}-\mathrm{Au}$ system at various (specified) beam kinetic energies, as obtained with either the 3-fluid model (left) or UrQMD (right) (adapted from Ref. [16]). The freeze-out boundary [17] is shown in the lower-left corner. 


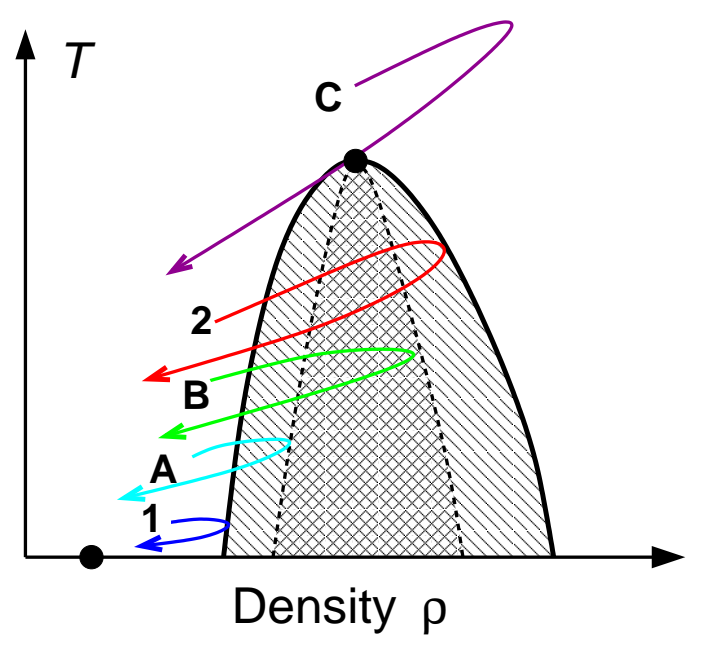

Figure 6: Illustration of how various ranges of the collision energy $E$ probe qualitatively different aspects of the equaiton of state: The phase coexistence region is not entered at all until $E>E_{1}$, the highest compression occurs inside the spinodal region for $E_{A}<E<E_{B}$, while it happens in the deconfined phase for $E>E_{2}$; the phase trajectory of the densest part goes right through the critical point at $E=E_{C}$. Thus most time is spent inside the spinodal region when $E$ is slightly below $E_{B}$.

Generally speaking, the prospects for spinodal decomposition can be expected to be better the more time the bulk of the matter spends inside the region of spinodal instability. Let us therefore consider how this aspect develops with the collision energy. For the following qualitative discussion, we assume that the phase diagram has the expected form with a first-order phase transition terminated by a critical point, as drawn schematically in Fig. 6 (see also Fig. 2). It seems natural to introduce a number of threshold values of the collision energy $E: E_{1}, E_{A}, E_{B}, E_{2}, E_{c}$. Their meaning is illustrated in Fig. 6 and they will be explained in turn below.

At the lowest collision energies, $E<E_{1}$, the compressions achieved are insufficient to bring any part of the matter inside the region of phase coexistence. Consequently, at such low energies, it would probably not be possible to probe the phase transition.

As the collision energy is raised above $E_{1}$, the phase trajectory $(\rho(t), T(t))$ of the most compressed matter makes ever larger incursions into the phase coexistence region. Characterizing such phase trajectories by the highest compression achieved, $\rho_{\max }(E)$, this "turning point" moves gradually across the phase coexistence region as $E$ is raised. It first enters the spinodal region for $E=E_{A}$ and it has traversed it fully for $E=E_{B}$, reaching the other side of the coexistence region at $E=E_{2}$.

At collision energies above $E_{2}$, the steady expansion of the bulk matter subsequent to its maximum compression drags the phase trajectory through the phase coexistence region (and the spinodal region inside it). The slope of the expansion phase trajectory steepens with $E$ (see Fig. 5) and the traversal time becomes steadily shorter, both because the expansion is faster and because the region of instability becomes narrower at the ever higher excitations encountered. At a certain "critical"collision energy, $E=E_{c}$, the phase trajectory passes right through the critical point $\left(\rho_{c}, T_{c}\right)$ and at supercritical collision energies, $E>E_{c}$, the phase trajectory will miss the unstable phase region altogether thus making any phase-transition signals increasingly unlikely to appear.

The evolving local thermodynamic conditions during a collision will generally differ from one location to another. Consequently, a single collision event gives rise to an entire bundle of phase trajectories and the above discussion pertains to just the phase trajectory of the most compressed matter of the collision system which, for a symmetric collision, is presumably located around the center. Furthermore, there is a dependence on the geometric features of the collision system, such as the nuclear sizes and the impact parameter. Thus the precise meaning of the various threshold 
energies is somewhat fuzzy and they play primarily a conceptual role. This underscores the fact that quantitative predictions must rely on detailed dynamical calculations.

Nevertheless, taking guidance from existing phase trajectories extracted from various transport simulations [16] (see Fig. 5), we have used the calculated growth rates to estimate the degree of amplification that might occur when the collision energy is adjusted to maximize the exposure to the spinodal instabilities. The resulting amplification amounts to one or two factors of $e$, which may suffice to trigger a phase separation due to the subsequent further amplification from the intermediate metastable phase region. While this conclusion gives grounds for guarded optimism, it also brings out the fact that a full dynamical simulation is needed for a more detailed assessment

This work was supported by the Director, Office of Energy Research, Office of High Energy and Nuclear Physics, Nuclear Physics Division of the U.S. Department of Energy under Contract No. DE-AC02-05CH11231. Helpful discussions with P.F. Bedaque, B. Friman, U. Heinz, V. Koch, K. Redlich, R. Sharma, W.J. Swiatecki, and D.N. Voskresensky are gratefully acknowledged.

\section{References}

[1] B. Borderie et al., Phys. Rev. Lett. 86, 3252 (2001).

[2] Ph. Chomaz, M. Colonna and J. Randrup, Phys. Reports 389, 263 (2004).

[3] D. Bower and S. Gavin, Phys. Rev. C 64, 051902 (2001).

[4] J. Randrup, Acta Phys. Hung. 22, 69 (2005).

[5] V. Koch, A. Majumder and J. Randrup, Phys. Rev. C 72, 064903 (2005).

[6] C. Sasaki, B. Friman and K. Redlich, Phys. Rev. Lett. 99, 232301 (2007).

[7] R.A.L. Jones, Soft Condensed Matter, Oxford University Press, 2002 [ISBN 0198505892].

[8] J. Randrup, Phys. Rev. C 79 (2009) 054911.

[9] V.V. Skokov and D.N. Voskresensky, nucl-th/0811-3868; nucl-th/0903-4335.

[10] D.G. Ravenhall, C.J. Pethick and J.M. Lattimer, Nucl. Phys. A 407, 571 (1983).

[11] R.W. Hasse and W.D. Myers, Geometrical Relationships of Macroscopic Nuclear Physics, Springer-Verlag, 1988 [ISBN 0-540-17510-5, 0-387-17510-5].

[12] W.D. Myers, W.J. Swiatecki and C.S. Wang, Nucl. Phys. A 436, 185 (1985).

[13] H. Heiselberg, C.J. Pethick and E.F. Staubo, Phys. Rev. Lett. 70, 1355 (1993).

[14] D.N. Voskresensky, M. Yasuhira and T. Tatsumi, Nucl. Phys. A 723, 291 (2003).

[15] J. Randrup, Phys. Rev. Lett. 92, 122301 (2004).

[16] I.C. Arsene et al., Phys. Rev. C 75, 034902 (2007).

[17] J. Randrup and J. Cleymans, Phys. Rev. C 74, 047901 (2006). 